\title{
AN ANALYSIS OF THE IMPORTANCE OF APPROPRIATE TIE BREAKING RULES IN DISPATCH HEURISTICS
}

\author{
Jorge M. S. Valente \\ Faculdade de Economia \\ Universidade do Porto \\ Porto - Portugal \\ jvalente@fep.up.pt \\ Recebido em 06/2005; aceito em 08/2005 \\ Received June 2005; accepted August 2005
}

\begin{abstract}
In this paper, we analyse the effect of using appropriate tie breaking criteria in dispatch rules. We consider four different dispatch procedures, and for each of these heuristics we compare two versions that differ only in the way ties are broken. The first version breaks ties randomly, while the second uses a criterion that incorporates problem-specific knowledge. The computational results show that using adequate tie breaking criteria improves the performance of the dispatch heuristics. The magnitude of the improvement is different for the four heuristics, and also depends on the characteristics of each specific instance. The use of problem-related knowledge for breaking ties should therefore be given some consideration in the implementation of dispatch rules.
\end{abstract}

Keywords: scheduling; dispatch rules; tie breaking.

\section{Resumo}

Neste artigo é analisado o efeito da utilização de regras de desempate apropriadas na eficácia de regras de despacho. São consideradas quatro regras de despacho diferentes, e para cada uma destas heurísticas são comparadas duas versões que diferem no modo como os empates são resolvidos. A primeira versão resolve os empates de forma aleatória, enquanto a segunda utiliza um critério que incorpora informação relativa ao problema em causa. Os resultados computacionais mostram que a utilização de critérios de desempate adequados melhora o desempenho das regras de despacho. A magnitude da melhoria é diferente para as quatro heurísticas, e depende igualmente das características específicas de cada instância. A utilização de informação relativa ao problema em causa para a resolução de empates deve assim ser considerada na implementação de regras de despacho.

Palavras-chave: sequenciamento; regras de despacho; resolução de empates. 


\section{Introduction}

In this paper, we analyse the importance of using appropriate tie breaking rules in constructive heuristics. More specifically, we consider the effect of adequate tie breaking criteria in the context of dispatch rules for scheduling problems. The issue of tie breaks, however, is also relevant for other types of constructive heuristics and other problems.

Dispatch rules are quite popular in scheduling, and a large number of papers in scheduling literature present or analyse dispatch heuristics. Also, dispatch rules are widely used in practice. In fact, most real scheduling systems are either based on dispatch rules, or at least use them to some degree. The book by Morton \& Pentico (1993) presents a detailed analysis of a large number of dispatching heuristics that are of interest to practitioners. The books by Pinedo (1995) and Pinedo \& Chao (1999) contain sections devoted to dispatching procedures, and also present examples of real scheduling systems that use dispatch rules. For large instances, dispatch rules are sometimes the only heuristic approach capable of generating solutions within reasonable computation times. Dispatch rules are also used by other heuristic procedures, e.g., they are often used to generate the initial sequence required by local search or metaheuristic algorithms.

Dispatch rules calculate a priority or urgency rating for each unprocessed job whenever a machine becomes available; the job with the largest priority is then selected for processing. However, ties may occur, i.e., there may exist several jobs with the same largest priority value. In this case, the tie has to be broken, and one of the tied jobs must be chosen. In several situations or implementations, these ties are broken at random.

In this paper, we analyse the effect of using appropriate tie breaking rules. We consider four dispatch rules (applied to three different scheduling problems), and compare two versions of these heuristics. These two versions differ in the way ties are broken: the first version breaks ties randomly, while the second breaks the ties using a criterion that incorporates problemspecific knowledge.

The remainder of the paper is organized as follows. In section 2, we describe the dispatch rules and the tie breaking criteria. The computational results are presented in section 3 . Finally, some concluding remarks are given in section 4.

\section{The Heuristic Procedures}

In this section, we present the dispatch rules that will be analysed, as well as the scheduling problems to which they are applied. We also describe the tie breaking criteria that uses problem-related information.

\subsection{The SPT heuristic}

The shortest processing time (SPT) rule was developed by Smith (1956), and sequences the jobs in non-decreasing order of their processing times. This dispatch rule optimally solves the single machine total completion time problem, and is used as a heuristic procedure for several other scheduling problems. In this paper, we consider using it as a heuristic for the single machine total tardiness problem. Formally, this problem can be described as follows. A set of $n$ independent jobs $\left\{J_{1}, J_{2}, \ldots, J_{n}\right\}$ has to be scheduled without preemptions on a 
single machine that can handle only one job at a time. The machine and the jobs are assumed to be continuously available from time zero onwards. Job $J_{j}, j=1,2, \ldots, n$, requires a processing time $p_{j}$ and has a due date $d_{j}$. For any given schedule, the tardiness of $J_{j}$ can be defined as $T_{j}=\max \left\{0, C_{j}-d_{j}\right\}$, where $C_{j}$ is the completion time of $J_{j}$. The objective is then to find a schedule that minimizes the total tardiness $\sum_{j=1}^{n} T_{j}$.

When all jobs are necessarily tardy (e.g., when all due dates are zero), the SPT rule gives an optimal sequence for the total tardiness problem. In fact, when all jobs are necessarily tardy, the total tardiness problem reduces to the total completion time problem, which, as previously mentioned, is optimally solved by the SPT rule. The SPT heuristic is also optimal if it results in a schedule with only tardy jobs. These results motivated the use of the SPT rule as a heuristic for the total tardiness problem, and it provides acceptable performance, particularly in situations where most jobs will be tardy.

The SPT rule in its original formulation only specifies that jobs should be scheduled in nondecreasing order of their $p_{j}$ s. Therefore, several ties may indeed occur. In the total completion time problem, ties can be broken arbitrarily without affecting the optimality of the SPT rule. In the total tardiness problem, however, an appropriate tie breaking criterion may be able to improve the effectiveness of the SPT heuristic. We consider using the jobs' due dates to break the ties: if two or more jobs have the same $p_{j}$, we select the job with the smallest $d_{j}$ to be processed next.

\subsection{The MDD heuristic}

The modified due date (MDD) heuristic was developed by Baker \& Bertrand (1982) for the total tardiness problem, and has also been used for several other scheduling problems. In this paper, we consider using it as a heuristic for the single machine total tardiness problem, just as originally proposed by Baker \& Bertrand (1982).

The original formulation of the MDD heuristic only specifies that, each time the machine becomes available, we select the job with the smallest modified due date $d_{j}^{\prime}=\max \left(t+p_{j}, d_{j}\right)$, where $t$ is the current time. Therefore, ties may occur. We use the jobs' processing times, and also the original due dates $d_{j}$ if necessary, to break the ties. When several jobs have the same modified due date, we select the job with the smallest $p_{j}$. If two or more jobs are still tied, we then choose the job with the lowest original due date.

\subsection{The EDD heuristic}

The earliest due date (EDD) rule was presented by Jackson (1955), and it sequences the jobs in non-decreasing order of their due dates. This rule gives an optimal sequence for the single machine maximum lateness problem, and is used as a heuristic for numerous other scheduling problems. We consider using it as a heuristic for the single machine total weighted tardiness problem. This problem is a generalization of the total tardiness problem. The problem formulation is similar, but now each job $J_{j}$ also has a tardiness penalty $w_{j}$, 
and the objective is to find a schedule that minimizes the total weighted tardiness $\sum_{j=1}^{n} w_{j} T_{j}$. The EDD rule is widely used as a heuristic for scheduling problems with a due date-related objective, and in the particular case of the single machine total weighted tardiness problem it is known that there exists an optimal solution in which non-late jobs are sequenced in EDD order.

The EDD rule in its original formulation only specifies that jobs should be scheduled in nondecreasing order of their $d_{j}$ s, so several ties may occur. In the maximum lateness problem, the optimality of the EDD rule is not affected by the way the ties are broken. In the total weighted tardiness problem, however, the results of the EDD rule might be improved by breaking ties according to some problem characteristics. We use the jobs' processing times, and also the tardiness weights if necessary, to break the ties. When several jobs have the same $d_{j}$, we select the job with the smallest $p_{j}$. If two or more jobs are still tied, we then choose the job with the largest $w_{j}$.

\subsection{The GreedyET heuristic}

The GreedyET heuristic was presented for the single machine early/tardy problem with no idle time by Valente \& Alves (2005). This greedy-type procedure is an adaptation of a heuristic originally introduced by Fadlalla, Evans \& Levy (1994) for the mean tardiness problem and adapted for the weighted tardiness problem by Volgenant \& Teerhuis (1999). The single machine early/tardy problem is a generalization of the total weighted tardiness problem. The characterization of the problem is similar, but in this case each job also has an earliness penalty $h_{j}$. For any given schedule, the earliness of $J_{j}$ can be defined as $E_{j}=\max \left\{0, d_{j}-C_{j}\right\}$. The objective is now to find a schedule that minimizes the total weighted earliness and tardiness $\sum_{j=1}^{n}\left(h_{j} E_{j}+w_{j} T_{j}\right)$, subject to the constraint that no idle time may be inserted in a schedule. This assumption that no machine idle time is allowed reflects a production setting where the cost of machine idleness is higher than the early cost incurred by completing any job before its due date, or the capacity of the machine is limited when compared with its demand, so that the machine must indeed be kept running.

The GreedyET heuristic can be described as follows. Let $t$ be the completion time of the current partial schedule and $c_{x y}$, with $x \neq y$, be the combined cost of scheduling jobs $J_{x}$ and $J_{y}$, in this order, in the next two positions in the sequence, i.e., $c_{x y}$ is the sum of the costs of $J_{x}$ and $J_{y}$ when they are completed at times $t+p_{x}$ and $t+p_{x}+p_{y}$, respectively. Let $L$ be a list with the indexes of the yet unscheduled jobs and $P(j)$ the priority of job $J_{j}$. The steps of the heuristic are:

Step 1: Initialize $L=\{1,2, \ldots, n\}$.

Step 2: $\operatorname{Set} P(j)=0$, for all $j \in L$.

Step 3: Determine $c_{i j}$ for all $i, j \in L, i \neq j$.

Step 4: For all pairs $\{i, j\} \in \mathrm{L}$, with $i \neq j$, do: 


$$
\begin{aligned}
& \text { If } c_{i j}<c_{j i}, \text { set } P(i)=P(i)+1 ; \\
& \text { If } c_{i j}>c_{j i} \text {, set } P(j)=P(j)+1 ; \\
& \text { If } c_{i j}=c_{j i} \text {, set } P(i)=P(i)+1 \text { and } P(j)=P(j)+1 .
\end{aligned}
$$

Step 5: Schedule job $J_{l}$ for which $P(l)=\max \{P(j) ; \mathrm{j} \in L\}$ and set $L=L \backslash\{l\}$.

Step 6: Stop if $\# L=1$; otherwise go to step 2 .

If $c_{i j}<c_{j i}$, it seems better to schedule job $J_{i}$ in the next position rather than job $J_{j}$. The priority $P(j)$ of job $J_{j}$ is therefore the number of times job $J_{j}$ is the preferred job for the next position when it is compared with all other unscheduled jobs. The GreedyET heuristic selects, at each iteration, the job with the highest priority $P(j)$. It is possible that ties occur, and the use of a problem-related criterion to break the ties may improve the performance of the GreedyET heuristic. When two or more jobs have the same $P(j)$ value, we break the ties using the EXPET dispatch rule priority function. The EXPET dispatch rule was developed by Ow \& Morton (1989) for the single machine early/tardy problem. An improved version of this dispatch rule has been presented by Valente \& Alves (2005), who also developed another dispatching procedure denoted as WPTMS. This version of the EXPET heuristic uses some functions for mapping several instance statistics into an appropriate value for a lookahead parameter that had previously been set at a fixed value (for further details concerning the EXPET rule, see Ow \& Morton (1989) and Valente \& Alves (2005)). When several jobs are tied, we calculate the EXPET urgency rating for each of those jobs, and the job with the largest rating is then selected for processing.

We remark that the computational tests performed by Valente \& Alves (2005) showed that the GreedyET heuristic is outperformed by both the EXPET and WPTMS dispatch rules. However, we selected this heuristic to illustrate the importance of adequate tie breaking rules for two reasons. First, the priority functions used in both the EXPET and WPTMS heuristics are piecewise-defined and have four different cases, which reduces the possibility for ties. Also, if a tie occurs for two or more tardy jobs (or jobs which are quite early), the priority values are such that the tie can be broken arbitrarily. Second, the greedy selection procedure used in the GreedyET heuristic is quite general, and may be applied to most combinatorial optimization problems. In fact, as previously mentioned, the same type of heuristic was previously applied to the total weighted and unweighted tardiness problems.

\section{Computational Results}

In this section, we present the results of the computational tests. A set of problems with 15 , $20,25,30,40,50,100,200,250,300,400,500$ and 1000 jobs was randomly generated as follows. For each job $J_{j}$, an integer processing time $p_{j}$, an integer tardiness penalty $w_{j}$ (required for both the total weighted tardiness and the early/tardy problems) and an integer earliness penalty $h_{j}$ (required for the early/tardy problem) were generated from one of the two uniform distributions $[1,10]$ and $[1,100]$, to create low and high variability, respectively. For each job $J_{j}$, an integer due date $d_{j}$ is generated from the uniform distribution $[P(1-T-R / 2), P(1-T+R / 2)]$, where $P$ is the sum of the processing time of all jobs, $T$ is the tardiness factor, set at $0.0,0.2,0.4,0.6,0.8$ and 1.0 , and $R$ is the range of due 
dates, set at $0.2,0.4,0.6$ and 0.8 . For each combination of instance size, processing time and penalty variability, $T$ and $R, 50$ instances were randomly generated. Therefore, a total of 1200 instances were generated for each combination of problem size and variability. All the algorithms were coded in Visual $\mathrm{C}++6.0$ and executed on a Pentium IV $1.7 \mathrm{GHz}$ personal computer. Due to the excessive computational times that would be required, the GreedyET heuristic was not applied to the instances with 1000 jobs. Throughout this section, and in order to avoid excessively large tables, we will present results only for some representative cases.

In Table 1, we present the average objective function value for both the heuristic versions that break the ties randomly (random), and the versions that use problem-related criteria (rule). We also give the relative improvement in the average objective function value (\%imp), calculated as (random - rule) / random $\times 100$. In Table 2, we give the number of instances for which the versions with a tie breaking rule performed better $(<)$, equal $(=)$ or worse $(>)$ than the versions with a random tie break. We also performed a test to determine if the difference between the two versions is statistically significant. Given that the two versions were used on exactly the same problems, a paired-samples test is appropriate. Since some of the hypotheses of the paired-samples t-test were not met, the non-parametric Wilcoxon test was selected. The significance values of this test, i.e., the confidence level values above which the equal distribution hypothesis is to be rejected, were always equal to 0.000 . From the results presented in these two tables, we can conclude the following. The versions that use a problem-related tie breaking criterion outperform the random versions, since their average objective function value is lower and they provide better results for a (sometimes quite) large number of the test instances. The Wilcoxon test values also indicate that the difference in distribution between the two versions is statistically significant.

The magnitude of the improvement provided by the rule-based versions, however, is quite different for the four dispatch rules. The SPT heuristic is the one that clearly benefits the most from the problem-based tie break criterion. The relative improvement in the average objective function value given by the rule version is much higher for this heuristic. For instances with a low processing time and penalty variability, the problem-based rule leads to a relative improvement of about $2.5 \%$, which is a most significant result when we consider the fact that it was achieved by a mere modification in the tie breaking criterion. Also, the rule version provides better results for most, and in several cases even all, of the test instances. The GreedyET and the MDD heuristics are the least affected by the problembased criterion. For these two heuristics, the improvement in the average objective function value is indeed marginal for the larger instances. Nevertheless, for the GreedyET heuristic, the number of instances for which a better result is obtained is larger than the number of times a worse result was given, although this difference is smaller than for the other heuristics. Also, the rule version of the MDD heuristic is quite rarely worse than the random version, and for some instance sizes with low variability it provides better results for a large number of instances. The EDD heuristic results are improved by the problem-related criterion for a large number of instances, and the relative improvement in the average objective function value is quite satisfactory for small to medium sized instances with low variability. 
Table 1 - Objective function value and relative improvement.

\begin{tabular}{|c|c|c|c|c|c|c|c|}
\hline \multirow[b]{2}{*}{ n } & \multirow[b]{2}{*}{ Heur } & \multicolumn{3}{|c|}{ low var } & \multicolumn{3}{|c|}{ high var } \\
\hline & & random & rule & \%imp & random & rule & \%imp \\
\hline \multirow[t]{4}{*}{15} & EDD & 1225 & 1210 & 1.23 & 104253 & 104072 & 0.17 \\
\hline & GreedyET & 1300 & 1297 & 0.25 & 99117 & 98799 & 0.32 \\
\hline & MDD & 163 & 162 & 0.26 & 1443 & 1443 & 0.02 \\
\hline & SPT & 188 & 184 & 2.05 & 1667 & 1664 & 0.19 \\
\hline \multirow[t]{4}{*}{25} & EDD & 3218 & 3193 & 0.80 & 272172 & 271964 & 0.08 \\
\hline & GreedyET & 3367 & 3360 & 0.20 & 262760 & 262171 & 0.22 \\
\hline & MDD & 417 & 417 & 0.15 & 3653 & 3652 & 0.02 \\
\hline & SPT & 491 & 480 & 2.24 & 4303 & 4292 & 0.25 \\
\hline \multirow[t]{4}{*}{50} & EDD & 12487 & 12427 & 0.48 & 1056227 & 1055693 & 0.05 \\
\hline & GreedyET & 12981 & 12963 & 0.14 & 999555 & 998756 & 0.08 \\
\hline & MDD & 1573 & 1571 & 0.08 & 14071 & 14070 & 0.01 \\
\hline & SPT & 1878 & 1832 & 2.46 & 16705 & 16662 & 0.26 \\
\hline \multirow[t]{4}{*}{100} & EDD & 48717 & 48602 & 0.24 & 4108916 & 4107824 & 0.03 \\
\hline & GreedyET & 50839 & 50818 & 0.04 & 3921300 & 3919602 & 0.04 \\
\hline & MDD & 6063 & 6060 & 0.06 & 53692 & 53689 & 0.01 \\
\hline & SPT & 7270 & 7090 & 2.49 & 64207 & 64041 & 0.26 \\
\hline \multirow[t]{4}{*}{250} & EDD & 302876 & 302585 & 0.10 & 25422942 & 25420286 & 0.01 \\
\hline & GreedyET & 313110 & 313021 & 0.03 & 24092620 & 24083087 & 0.04 \\
\hline & MDD & 37494 & 37483 & 0.03 & 328629 & 328621 & 0.00 \\
\hline & SPT & 45126 & 43990 & 2.52 & 395929 & 394868 & 0.27 \\
\hline \multirow[t]{4}{*}{500} & EDD & 1206134 & 1205544 & 0.05 & 101835682 & 101830330 & 0.01 \\
\hline & GreedyET & 1240704 & 1240419 & 0.02 & 95300548 & 95294007 & 0.01 \\
\hline & MDD & 148075 & 148057 & 0.01 & 1310519 & 1310501 & 0.00 \\
\hline & SPT & 179017 & 174423 & 2.57 & 1580558 & 1576371 & 0.26 \\
\hline \multirow[t]{3}{*}{1000} & EDD & 4832414 & 4831238 & 0.02 & 406323498 & 406312563 & 0.00 \\
\hline & MDD & 592850 & 592816 & 0.01 & 5218427 & 5218388 & 0.00 \\
\hline & SPT & 716824 & 698381 & 2.57 & 6301302 & 6284376 & 0.27 \\
\hline
\end{tabular}


Table 2 - Objective function value comparison.

\begin{tabular}{|c|c|c|c|c|c|c|c|}
\hline \multirow[b]{2}{*}{$\mathbf{n}$} & \multirow[b]{2}{*}{ Heur } & \multicolumn{3}{|c|}{ low var } & \multicolumn{3}{|c|}{ high var } \\
\hline & & $<$ & $=$ & $>$ & $<$ & $=$ & $>$ \\
\hline \multirow[t]{4}{*}{15} & EDD & 462 & 627 & 111 & 92 & 1078 & 30 \\
\hline & GreedyET & 158 & 951 & 91 & 161 & 963 & 76 \\
\hline & MDD & 98 & 1102 & 0 & 15 & 1184 & 1 \\
\hline & SPT & 632 & 568 & 0 & 94 & 1106 & 0 \\
\hline \multirow[t]{4}{*}{25} & EDD & 564 & 528 & 108 & 131 & 1026 & 43 \\
\hline & GreedyET & 216 & 879 & 105 & 233 & 845 & 122 \\
\hline & MDD & 112 & 1088 & 0 & 19 & 1181 & 0 \\
\hline & SPT & 940 & 260 & 0 & 265 & 935 & 0 \\
\hline \multirow[t]{4}{*}{50} & EDD & 702 & 418 & 80 & 207 & 925 & 68 \\
\hline & GreedyET & 313 & 701 & 186 & 389 & 586 & 225 \\
\hline & MDD & 184 & 1016 & 0 & 36 & 1164 & 0 \\
\hline & SPT & 1149 & 51 & 0 & 656 & 544 & 0 \\
\hline \multirow[t]{4}{*}{100} & EDD & 758 & 400 & 42 & 368 & 742 & 90 \\
\hline & GreedyET & 459 & 463 & 278 & 514 & 350 & 336 \\
\hline & MDD & 332 & 868 & 0 & 62 & 1138 & 0 \\
\hline & SPT & 1192 & 8 & 0 & 1015 & 185 & 0 \\
\hline \multirow[t]{4}{*}{250} & EDD & 806 & 378 & 16 & 574 & 513 & 113 \\
\hline & GreedyET & 507 & 290 & 403 & 657 & 116 & 427 \\
\hline & MDD & 525 & 675 & 0 & 112 & 1088 & 0 \\
\hline & SPT & 1200 & 0 & 0 & 1191 & 9 & 0 \\
\hline \multirow[t]{4}{*}{500} & EDD & 823 & 371 & 6 & 652 & 466 & 82 \\
\hline & GreedyET & 546 & 204 & 450 & 677 & 77 & 446 \\
\hline & MDD & 668 & 532 & 0 & 204 & 996 & 0 \\
\hline & SPT & 1200 & 0 & 0 & 1200 & 0 & 0 \\
\hline \multirow[t]{3}{*}{1000} & EDD & 824 & 372 & 4 & 725 & 434 & 41 \\
\hline & MDD & 627 & 573 & 0 & 286 & 914 & 0 \\
\hline & SPT & 1200 & 0 & 0 & 1200 & 0 & 0 \\
\hline
\end{tabular}


The number of instances for which the rule-based versions give better results increases with the instance size. However, the relative improvement decreases as $n$ increases for the MDD, EDD and GreedyET heuristics, while it tends to increase for the SPT dispatch rule. This latter result concerning the SPT rule is not surprising. In fact, as the instance size increases, the number of jobs sharing the same processing time also increases. Therefore, more ties will take place, and the extra information given by the problem-related criterion is used a larger number of times. The variability of the processing times and penalties does not seem to have much effect on the GreedyET heuristic, though it significantly affects the other procedures. For the MDD, SPT and EDD heuristics, both the relative improvement and the number of instances for which a better result is obtained are much higher when the variability is low. This effect of the processing times and penalties variability is to be expected. When the variability of the processing times is low, more jobs share the same $p_{j}$, and therefore more ties will occur in the SPT dispatch rule. Also, when the processing time variability is low, the intervals in which the due dates are contained are tighter, since $P$, the sum of the $p_{j} \mathrm{~s}$, is smaller. The probability of two jobs having identical due dates is then higher, and more ties will take place in the EDD heuristic. The number of ties will also be larger in the MDD heuristic when the probability of two jobs having identical due dates and processing times is higher.

The effect of the $T$ and $R$ parameters on the relative objective function value improvement is given in Table 3 for instances with 100 jobs. From the results presented in this table, we can see that the tardiness factor $T$ has a significant effect on the relative improvement provided by the rule-based tie break criterion. In the case of the GreedyET heuristic, the relative improvement is higher for instances with a tardiness factor of 0.4 or 0.6 . The improvement decreases as $T$ approaches its extreme values, and is mostly inexistent when $T$ is equal to 0.0 or 1.0. This result is to be expected, since the early/tardy problem is more difficult for the intermediate $T$ values. If all jobs are necessarily early (late), an optimal schedule can easily be obtained, and when $T=0.0$ (1.0), most jobs will indeed be early (late). For the intermediate $T$ values, there is a greater balance between the number of early and tardy jobs, and the problem becomes more difficult. Therefore, there are more possibilities for improvement when $T$ is equal to 0.4 or 0.6 (i.e., when the problem is harder).

For the MDD, SPT and EDD heuristics, the relative improvement is lower when $T=1.0$, and then gradually increases as $T$ decreases towards 0.2 . For some parameter combinations, namely for $T=0.0$, or when $T=0.2$ and $R$ is equal to 0.6 or 0.8 , the optimal solution for most or all instances of the weighted and unweighted tardiness problems has no tardy jobs, and therefore the optimal objective function value is zero. The --- sign in the MDD and EDD results corresponding to these parameter values means that both versions of these heuristics obtained such an optimal solution for all instances with these parameter combinations. The relative improvement values of the SPT heuristic are also quite high for these same parameter combinations. These values are somewhat misleading, since they correspond to situations where the average objective function value is quite low for both versions. The decrease in the total tardiness made possible by the rule-based criterion is therefore small when measured in absolute terms. When $T=1.0$, most jobs will be tardy, and both the weighted and unweighted tardiness problems are easy to solve. As $T$ decreases, the proportion of tardy jobs decreases, and the problem becomes harder. Hence, there are more possibilities for improvement as $T$ decreases (i.e., as the problem becomes harder), and the relative improvement provided by the problem-based tie break criterion correspondingly increases. 
Table 3 - Relative improvement for instances with 100 jobs.

\begin{tabular}{|c|c|c|c|c|c|c|c|c|c|}
\hline \multirow[b]{2}{*}{ Heur } & \multirow[b]{2}{*}{$\mathbf{T}$} & \multicolumn{4}{|c|}{ low var } & \multicolumn{4}{|c|}{ high var } \\
\hline & & $\mathrm{R}=0.2$ & $\mathrm{R}=0.4$ & $R=0.6$ & $\mathrm{R}=0.8$ & $\mathrm{R}=0.2$ & $\mathrm{R}=0.4$ & $R=0.6$ & $\mathrm{R}=0.8$ \\
\hline \multirow[t]{6}{*}{ EDD } & 0.0 & --- & --- & --- & --- & --- & --- & --- & --- \\
\hline & 0.2 & 2.14 & 2.61 & --- & --- & 0.27 & 0.74 & --- & --- \\
\hline & 0.4 & 0.90 & 0.67 & 0.70 & 1.71 & 0.13 & 0.13 & 0.12 & 0.14 \\
\hline & 0.6 & 0.51 & 0.34 & 0.29 & 0.29 & 0.05 & 0.03 & 0.04 & 0.03 \\
\hline & 0.8 & 0.43 & 0.26 & 0.17 & 0.12 & 0.03 & 0.03 & 0.03 & 0.01 \\
\hline & 1.0 & 0.31 & 0.13 & 0.11 & 0.07 & 0.03 & 0.02 & 0.01 & 0.01 \\
\hline \multirow[t]{6}{*}{ GreedyET } & 0.0 & 0.00 & 0.00 & 0.01 & 0.02 & -0.01 & 0.00 & 0.00 & 0.00 \\
\hline & 0.2 & 0.04 & 0.17 & 0.07 & 0.17 & 0.00 & -0.05 & 0.07 & 0.11 \\
\hline & 0.4 & 0.15 & 0.22 & 0.14 & 0.37 & 0.19 & 0.23 & -0.05 & 0.25 \\
\hline & 0.6 & 0.22 & 0.03 & -0.29 & 0.51 & 0.21 & 0.29 & 0.56 & 0.52 \\
\hline & 0.8 & 0.03 & 0.09 & -0.01 & 0.02 & 0.07 & 0.03 & 0.03 & 0.02 \\
\hline & 1.0 & 0.00 & 0.00 & 0.00 & 0.00 & 0.00 & 0.00 & 0.00 & 0.00 \\
\hline \multirow[t]{6}{*}{ MDD } & 0.0 & --- & --- & --- & --- & --- & --- & --- & --- \\
\hline & 0.2 & 0.57 & 0.00 & --- & --- & 0.21 & 0.00 & --- & --- \\
\hline & 0.4 & 0.27 & 0.28 & 0.38 & 0.08 & 0.01 & 0.00 & 0.00 & 0.18 \\
\hline & 0.6 & 0.28 & 0.18 & 0.17 & 0.13 & 0.02 & 0.04 & 0.01 & 0.02 \\
\hline & 0.8 & 0.21 & 0.04 & 0.04 & 0.01 & 0.00 & 0.01 & 0.00 & 0.00 \\
\hline & 1.0 & 0.00 & 0.00 & 0.00 & 0.00 & 0.00 & 0.00 & 0.00 & 0.00 \\
\hline \multirow[t]{6}{*}{ SPT } & 0.0 & 98.84 & 76.67 & 50.67 & 42.13 & 16.18 & 5.50 & 5.80 & 4.43 \\
\hline & 0.2 & 11.55 & 21.63 & 22.63 & 20.48 & 1.41 & 2.11 & 2.40 & 2.54 \\
\hline & 0.4 & 2.97 & 5.69 & 8.30 & 10.34 & 0.32 & 0.63 & 0.90 & 0.93 \\
\hline & 0.6 & 1.38 & 2.96 & 4.04 & 4.85 & 0.12 & 0.33 & 0.46 & 0.58 \\
\hline & 0.8 & 0.77 & 1.61 & 2.09 & 2.51 & 0.09 & 0.16 & 0.18 & 0.25 \\
\hline & 1.0 & 0.22 & 0.45 & 0.55 & 0.79 & 0.02 & 0.05 & 0.06 & 0.09 \\
\hline
\end{tabular}

The runtimes were virtually identical for both versions of the heuristics, and for that reason they will not be presented. The use of appropriate tie breaking criteria in dispatch rules is therefore recommended, since they can improve the heuristic results with no noticeable additional computational effort. The magnitude of the improvement provided by a problembased criterion will most likely depend, as these computational results have shown, both on the specific heuristic and on the characteristics of each particular instance. 


\section{Conclusion}

In this paper, we analysed the effect of using appropriate tie breaking criteria in dispatch rules for scheduling problems. Four different dispatch procedures were considered, and for each of these heuristics we compared two versions that differ only in the way ties are broken. The first version breaks ties randomly, while the second uses a criterion that incorporates problem-specific knowledge.

The computational results show that using adequate tie breaking criteria improves the performance of the dispatch heuristics, with virtually no additional computational effort. The magnitude of the improvement, however, was quite different for the four heuristics. The improvement also depends on the characteristics of each specific instance. As expected, the improvement is more significant for instances where the probability of occurring ties is higher. The effectiveness of the problem-based tie breaking criteria is also greater for the more difficult instances, where there are more possibilities for improvement. The use of problem-related knowledge for breaking ties should therefore be given some consideration in the implementation of dispatch rules, particularly when they are used in production scheduling systems.

\section{Acknowledgement}

The author would like to thank the anonymous referees for several helpful comments and suggestions that were used to improve this paper.

\section{References}

(1) Baker, K. R. \& Bertrand, J. W. M. (1982). A dynamic priority rule for scheduling against due dates. Journal of Operations Management, 3, 37-42.

(2) Fadlalla, A.; Evans, J. R. \& Levy, M. S. (1994). A greedy heuristic for the mean tardiness sequencing problem. Computers \& Operations Research, 21, 329-336.

(3) Jackson, J. R. (1955). Scheduling a production line to minimize maximum tardiness. Management Science Research Project, Research Report 43, University of California, Los Angeles.

(4) Morton, T. E. \& Pentico, D. (1993). Heuristic Scheduling Systems. Wiley, New York.

(5) Ow, P. S. \& Morton, E. T. (1989). The single machine early/tardy problem. Management Science, 35, 177-191.

(6) Pinedo, M. (1995). Scheduling: Theory, Algorithms and Systems. Prentice Hall, New Jersey.

(7) Pinedo, M. \& Chao, X. (1999). Operations Scheduling with Applications in Manufacturing and Services. McGraw-Hill, Singapore.

(8) Smith, W. E. (1956). Various optimizers for single-stage production. Naval Research Logistics Quarterly, 3, 59-66. 
(9) Valente, J. M. S. \& Alves, R. A. F. S. (2005). Improved heuristics for the early/ tardy scheduling problem with no idle time. Computers \& Operations Research, 32, 557-569.

(10) Volgenant, A. \& Teerhuis, E. (1999). Improved heuristics for the n-job single-machine weighted tardiness problem. Computers \& Operations Research, 26, 35-44. 